\title{
Dietary shifts and social interactions drive temporal fluctuations of the gut microbiome from wild redfronted lemurs
}

\author{
Tatiana Murillo (iD ${ }^{1,2}$, Dominik Schneider $\mathbb{D}^{2}$, Claudia Fichtel $\mathbb{D}^{1,3}$ and Rolf Daniel (iD ${ }^{2,3}$ 凶 \\ (c) The Author(s) 2022
}

\begin{abstract}
Animals living in highly seasonal environments adapt their diets accordingly to changes in food availability. The gut microbiome as an active participant in the metabolization of the host's diet should adapt and change with temporal diet fluctuations, but dietary shifts can be short-term and, hence, difficult to detect in cross-sectional studies. Therefore, we performed a longitudinal study combining repeated sampling of fecal samples with observations of feeding behavior in wild redfronted lemurs. We amplified taxonomical marker genes for assessing the bacteria, archaea, protozoa, helminths, and fungi, as well as the active bacterial community inhabiting their gut. We found that the most abundant protozoans were Trichostomatia and Trichomonadida, and the most abundant helminths were Chromadorea. We detected known members of the gut mycobiome from humans but in low abundances. The archaeal community is composed only of members of Methanomethylophilaceae. The predominant phyla in the entire bacterial community were Bacteroidota and Firmicutes while the most abundant genera harbor so far unknown bacteria. Temporal fluctuations at the entire community level were driven by consumption of fruits and flowers, and affiliative interactions. Changes in alpha diversity correlated only with the consumption of flowers and leaves. The composition of the entire and active bacterial community was not significantly different, but the most abundant taxa differed. Our study revealed that monthly changes in the bacterial community composition were linked to fruit and flower consumption and affiliative interactions. Thus, portraying the importance of longitudinal studies for understanding the adaptations and alterations of the gut microbiome to temporal fluctuations.
\end{abstract}

ISME Communications; https://doi.org/10.1038/s43705-021-00086-0

\section{INTRODUCTION}

The gut microbiome is a complex fluctuating microbial ecosystem comprising prokaryotic and eukaryotic microorganisms playing a pivotal role in immunity, physiology, metabolism, and susceptibility to disease of the host [1,2]. Investigations of factors driving these fluctuations help to understand how this ecosystem adapts to the changing conditions, and the potential effects these variations have on the health and fitness of their hosts [2-4].

Essential nutrient cycling processes of the gut ecosystem occur between the host diet, the microorganisms, and the host itself $[1,2,4]$. Bacteria catalyze the fermentation of dietary fiber and starch into short-chain fatty acids and monosaccharides taken up by the host and other microorganisms [1, 2]. They also provide ammonia for protein synthesis by metabolizing essential and nonessential amino acids [2]. The host diet shapes the microbial gut communities and the presence of certain microorganisms is crucial for proper degradation and uptake of nutrients from diet and the resilience of the gut ecosystem $[1,2,4]$. Therefore, the gut microbial ecosystem of wild animals living in highly seasonal environments should be capable of adapting to dietary changes following fluctuations in food availability and seasonality [3-5]. Research in wild mice (Apodemus sylvaticus), Tibetan macaques
(Macaca thibetana) and pandas (Ailuropoda melanoleuca) found marked seasonal variations in the gut microbiome composition and diversity associated with environmental fluctuations affecting food availability [5-7]. Furthermore, cross-sectional studies in black howler monkeys (Alouatta pigra), white faced capuchins (Cebus capucinus), and Verreaux's sifakas (Propithecus verreauxi) determined these fluctuations correlate with changes in foraging and feeding behaviors [8-10]. Nonetheless, by sampling only representative months of each season, short-term dietary and gut microbiome shifts might be undetected [4]. In the Hadza huntergatherers, a seasonal cycling of the gut microbiome following seasonal changes in their diets between fruit foraging and hunting was detected [11]. Longitudinal studies in great apes (Gorilla gorilla gorilla, Gorilla beringei beringei and Pan troglodytes troglodytes) and geladas (Theropithecus geladas) determined seasonal fluctuations of the gut microbiome correlate with rainfall, temperature, and food availability $[12,13]$. While a time series study in baboons (Papio cynocephalus) detected a highly dynamic gut microbiome varying according to the group's diet, rainfall, and the quality of the water sources [14]. Thus, enhancing the importance of time series analysis in wild animals to determine how the gut microbial communities adapt to seasonal changes $[4,8,12,14]$.

\footnotetext{
${ }^{1}$ Behavioral Ecology and Sociobiology Unit, German Primate Center, Göttingen, Germany. ${ }^{2}$ Genomic and Applied Microbiology and Göttingen Genomics Laboratory, Institute of Microbiology and Genetics, Georg-August-University of Göttingen, Göttingen, Germany. ${ }^{3}$ These authors contributed equally: Claudia Fichtel, Rolf Daniel.

然mail: rdaniel@gwdg.de
} 
To our knowledge, all taxonomic profiling studies in wild animals focus on the amplification of 16S rRNA gene from DNA, hence studying the entire community. This approach can be biased by the number of $16 \mathrm{~S}$ rRNA operons and the presence of dormant or dead cells in the sample [15]. Conversely, when amplifying the $16 \mathrm{~S}$ rRNA transcripts, only the bacterial community that is actively replicating is investigated, providing insights into the potentially active community [16]. This approach can provide better proxies into the functional metabolic changes that the gut microbiome undergoes as a response to seasonality $[2,4]$.

We performed a longitudinal analysis of the entire and active gut bacterial community in a wild primate, the redfronted lemur (Eulemur rufifrons). Their habitat, Kirindy Forest in Madagascar, is highly seasonal having a cold dry season from April to October and a warm rainy season from November to March [17, 18]. These environmental conditions cause changes in the availability of food and water sources, posing adaptive challenges for these animals [1921]. Redfronted lemurs are mainly frugivorous but consume leaves, and flowers following seasonal fluctuations, and adjust their drinking behavior according to the available water sources [20,21]. Hence, these redfronted lemurs are a suitable study system to characterize temporal fluctuations in the gut microbiome composition. Moreover, they possess a high eukaryotic parasite richness with variations in their monthly prevalence as detected from morphological studies, suggesting complex prokaryotic and eukaryotic interactions occur in their guts [18, 22, 23]. However, their gut mycobiome is still unexplored despite its potential metabolic importance [24, 25].

For 1 year, we collected up to three fecal samples per month for each individual and conducted regular animal focal observations to determine their dietary composition and affiliative interactions. Since previous research suggested that social group and home range can also impact the gut microbiome $[10,26]$, we studied only one group consisting of five individuals to control for these potential confounding factors. To characterize the microbiome composition, we assessed the entire and active bacterial community as well as other inhabitants of the gut, including Protozoa, helminths, Fungi, and Archaea. We hypothesize that by using a longitudinal approach, we [1] determine temporal fluctuations in composition and diversity of the bacterial entire and active community correlate to monthly changes in diet and affiliative interactions, [2] find no significant differences between the entire and the active bacterial communities, and [3] detect temporal changes in the abundances of the eukaryotic community.

\section{METHODS \\ Sample, behavioral, and environmental data collection}

This study was conducted at the research station of the German Primate Center in Kirindy Forest, Western Madagascar $\left(44^{\circ} 39^{\prime} \mathrm{E}, 20^{\circ} 03^{\prime} \mathrm{S}\right)$ from May 2018 to April 2019 [17]. Samples and data were collected over 1 year from five redfronted lemurs belonging to the same group; three adult females (FLucF, FTorF, and FMayF), one juvenile female (FBonF) and one adult male (FCaiM) (Supplementary Table S1). Fecal samples were collected in RNAlater (Thermofisher Scientific, Massachusetts, USA) from the forest floor immediately after defecation between $7: 30$ and 11:00, stored at $-20^{\circ}$ $\mathrm{C}$ in the field station and later at $-80^{\circ} \mathrm{C}$ in Germany. A total of 142 samples were collected, with an average of two samples per individual per month (Supplementary Table S1). Behavioral data was collected by continuous focal observations for $30 \mathrm{~min}$ in the morning $(7: 30-11: 00)$ and afternoon (14:00-17:00). Feeding behaviors were recorded by protocolling their duration and the ingested food item (leaves, flowers, or fruits). For affiliative interactions, we protocolled the duration of grooming and body contact behavior. Environmental data (daily temperature and precipitation) were collected at the field station with a Tropos data logger (Lambrecht meteo, Göttingen, Germany).

DNA extraction and amplification of taxonomic marker genes DNA extractions were performed with the PowerSoil DNA isolation kit (Qiagen, Hilden, Germany) using $150 \mathrm{mg}$ fecal sample following the manufacturer's instructions but including a bead beating step of $6.5 \mathrm{~m} / \mathrm{s}$ and $24 \times 2$ for $20 \mathrm{~s}$ using FastPrep- $24^{\mathrm{TM}} 5 \mathrm{G}$ (MP Biomedicals, California, USA). PCR reactions for all taxonomical marker genes were performed in triplicates with the primers and thermocycling protocols listed in the Supplementary Table S2 and included a negative control without DNA template and a positive control [27-32]. Triplicates per sample were pooled equimolar, purified, and sequenced as in [33].

\section{RNA extraction and cDNA synthesis}

RNA was extracted from $250 \mathrm{mg}$ fecal sample using the RNeasy Power Microbiome kit (Qiagen) following the manufacturer's instructions, and according to the protocol from [33].

\section{Bioinformatic processing of amplicon data}

Paired-end reads were quality-filtered with Fastp0.20.0 [34] using default settings with the addition of an increased per base phred score of 20, base pair corrections by overlap (-c), as well as $5^{\prime}$ - and $3^{\prime}$-end read-trimming with a sliding window of 4, a mean quality of 20 and minimum sequence length of 50 bp. Quality-controlled reads were merged with PEARv0.9.11 [35] and primer-clipping was performed with cutadapt2.5 [36] with default settings. VSEARCH2.14.1 [37] was used for size-sorting, size-filtering ( $16 \mathrm{~S}$ rRNA $\geq 300$ bp; $18 \mathrm{~S}$ rRNA $\geq 250 \mathrm{bp}$; ITS $2 \geq 140 \mathrm{bp}$ ) and dereplication. The sequences were denoised with UNOISE3 [38] using default settings and chimeras were removed with UCHIME3 (de novo followed by reference-based) [39] leading to the final set of amplicon sequence variants (ASVs). Then all reads were mapped against the ASVs and taxonomy was assigned with a minimum identity of $90 \%$ using BLAST2.9.0 + [40] against different databases according to the taxonomical marker gene. The databases were SILVA SSU 138 NR [41] for $16 \mathrm{~S}$ rRNA, PR ${ }^{2}$ SSU rRNA [42] for $18 \mathrm{~S}$ rRNA and UNITE 8.2 [43] for ITS2. Best hits were only accepted if $\left(\frac{\% \text { identity }+\% \text { coverage }}{2}\right) \geq 93$ following the recommendation of SILVA database [41]. Best blastn hit identity for bacterial species $<98.7 \%$ or genus $<94.5 \%$ were corrected to unclassified [44]. Functional predictions were performed using Faprotax1.2.3 [45] for the bacterial $16 \mathrm{~S}$ rRNA gene data after beforementioned filters were applied. All sequencing statistics are presented in Supplementary Table S3.

\section{Data visualization and statistical analysis}

Data visualization and statistical analysis were performed using Rv3.6.2 [46] and RStudiov1.20.5033 [47] by using the packages ampvis2 [48], ape [49], stringr [50], reshape2 [51], viridis, data.table [52], tidyverse [53], and ggplot2 [54]. Datasets for barcharts, heatmaps, and linecharts were normalized using GMPR [55], whereas data was rarefied for diversity and multivariate analysis (Supplementary Table S3). A phylogenetic tree was generated by aligning all sequences with MAFFTv7.407-1 [56] at 100 iterations, calculated using FastTreeMPv2.1.7 [57] and midpoint-rooted using FigTree v1.4.4 [58] for estimating Faith's phylogenetic diversity (PD) with the package picante [59].

For the $18 \mathrm{~S}$ rRNA gene amplicon analysis of eukaryotic parasites and symbionts, samples with $<9000$ reads were excluded leaving 115 samples. ASVs from the kingdoms previously reported as inhabitants of the gastrointestinal tract of animals: Cercozoa, Ciliophora, Metazoa, Apicomplexa, Lobosa, Conosa, and Metamonada were analyzed [23, 60]. For the ITS2 dataset samples with $<7000$ reads after quality-filtering samples were removed leaving 125 samples for analysis.

ANCOM analysis to estimate differential taxa between seasons. To determine bacterial genera with significant different relative abundances between seasons, we used ANCOM 2.1 [61] and the packages exactRankTests [62], nlme [63], compositions [64], and readr [65] by using the repeated measures model with season as main variable and individual as random effect, and 0.7 as threshold of the $W$ statistic.

Multivariate analysis to study temporal changes in $\beta$-diversity. Principal coordinate analyses (PCOA) using weighted UniFrac distances (WUnifrac) $[66,67]$ were calculated in ampvis2 [48]. To test for correlations of the behavioral and environmental variables an environmental fit with 999 permutations was calculated and corrected for repeated sampling by using strata as individual with vegan [66]. A PERMANOVA test was calculated with the adonis function from the vegan package to test for significant differences between individual $\beta$-diversity calculated as WUnifrac. Mantel tests using Spearman correlations were calculated with the vegan package to estimate correlations between $\beta$-diversity from WUniFrac distances and time between sample collection. 
Linear mixed model for estimating the effects on bacterial composition. The effects of feeding behaviors and affiliative interactions on the bacterial composition of the entire bacterial community were tested by fitting a Linear Mixed Model (LMM) with Ime4 [68]. The model included monthly feeding rates ( $\mathrm{min} / \mathrm{h}$ ) on fruits, leaves, or flowers, and affiliative interactions $(\mathrm{min} / \mathrm{h})$ per individual as test predictors, and mean monthly precipitation $(\mathrm{mm})$ as control predictor. Taxa with abundances $<0.5 \%$ in a sample were removed to account for index hopping during sequencing [69]. To deal with data compositionality, the microbial proportions of each sample were centered log-ratio transformed [70]. The random intercepts effects of individual, taxon, sample, and taxon nested within individual (taxonindividual) were included, the latter to account for individual specific microbial compositions. Random slopes for all predictors in taxon, individual, and taxon-individual were included, excluding flower feeding rates for taxon-individual [71]. Parameters for the correlations between random intercepts and slopes within taxon and taxon-individual were included [71] but not within individual because they were unidentifiable [72]. Assumptions of normally distributed and homogeneous residuals were checked visually with QQ-plots of residuals and residuals plotted against fitted values which revealed no obvious deviations. No issues of collinearity were detected by calculating Variance Inflation Factors using car [73] on a model lacking the random effects (maximum: 1.203). The crucial terms in this model were the random slopes within taxon representing the taxon-specific effects of the test predictors and were tested with a permutation test by shuffling the labels of taxa within sample $[74,75]$. As a test statistic, we used the difference between the log likelihoods of the full model and simpler models. One of the simpler models lacked all random slopes within the sample except that of precipitation allowing a full-null model comparison by testing the combined effects of all test predictors. The others lacked the individual random slopes (except precipitation) within taxon allowing to test their individual contribution. A total of 1000 permutations including the original data as one permutation were conducted, and $p$-values were calculated as the proportion of permutations that revealed a test statistic at least as large as that of the original data. If an individual random slope effect was significant, then the effect of the respective predictor differs between taxa. The 20 taxa differing most from the average effect across all taxa, meaning they had the largest absolute values of the respective Best Linear Unbiased Predictors (BLUPs), were inspected [76]. Model stability was assessed by dropping individuals one at time, fitting the full model to each of the subsets, and then comparing the estimates derived with those obtained for the full model revealing it was acceptable. Residuals for each combination of taxon and predictor were plotted verifying the presence of linear trends.

Linear mixed models for estimating effects on alpha diversity. The effects of feeding behaviors and affiliative interactions on alpha diversity for the entire and active bacterial community were estimated by fitting a LMM using Ime4 [68], MuMIn [77], and visualized with sjPlot [78]. The response variable was $P D$, which was log-transformed for the model of the active community. Affiliative interactions were log-transformed to achieve a more symmetrical distribution and avoid influential cases, and all predictors were z-transformed to facilitate model convergence. We included individual identity as a random intercept effect and the random slopes of all fixed effects into individual identity to keep the type I error at the nominal level of $5 \%$ [71]. For estimating the significance of the test predictors, a null model excluding the test predictors was calculated and then compared to the full model using a likelihood ratio test. We determined the effect of single fixed effects using likelihood ratio tests comparing the full model with reduced models removing one fixed effect at a time [71]. Model assumptions and collinearity (DNA: 1.203; RNA: 1.205) were checked as in the LMM for bacterial composition with no obvious deviations from these assumptions. Model stability was assessed as described above.

Procrustes analysis. Procrustes analysis and significance testing with protest were performed using vegan [66] to test for correlations between the plant material detected from the $18 \mathrm{~S}$ rRNA gene amplicons and the entire bacterial community from calculated PCoAs of Bray Curtis dissimilarity matrices in ampvis2 [48]. Only those samples with $>1000$ reads for Archaeplastida were analyzed, leaving 97 samples after rarefaction. The same test was used to determine significant differences between the composition of the entire and active bacterial community from the PCoAs from WUnifrac distances. A summary of all statistical results is depicted in Supplementary Table S4.
Gene alignments and phylogenetic tree from eukaryotic data Sequence alignments were done with MUSCLE [79] with UPGMA and default settings. Phylogenetic trees were calculated with the Maximum Likelihood method, Tamura-Nei model, and 1000 bootstrap in MEGA X [80]. The 18S rRNA gene and ITS2 sequences from representative nematodes and Fungi were retrieved from GenBank database [81].

\section{Data deposition}

The $16 \mathrm{~S}$ rRNA gene and transcripts, $18 \mathrm{~S}$ rRNA gene, and ITS2, paired-end raw reads were deposited in the National Center for Biotechnology Information Sequence Read Archive (SRA) under the Bioproject PRJNA694983. SRA numbers are in Supplementary Table S1.

\section{RESULTS}

\section{Composition of the redfronted lemur gut microbiome}

The most abundant bacterial phyla in the five redfronted lemurs were constant throughout the sampling period with varying relative abundances; these were Bacteroidota $(30.6 \% \pm 7.6)$, Firmicutes $(30.0 \% \pm 8.2)$, Proteobacteria $(12.3 \% \pm 6.5)$, Spirochaetota $(8.7 \% \pm 2.5)$ and Verrucomicrobiota $(6.3 \% \pm 2.2)$ (Fig. $1 \mathrm{~A}$ and Supplementary Table S5). These were consistent for all individuals exempting an increase of Firmicutes (55.7\%) and Proteobacteria in February for FBonF, and an increase of Fusobacteriota (9.9\%) in January for FLucF.

The taxa detected from the amplification of the $18 \mathrm{~S}$ rRNA gene were Metazoa $(56.9 \% \pm 22.7)$, Streptophyta $(21.3 \% \pm$ 13.2), Fungi $(6.6 \% \pm 6.1)$, Ciliophora $(9.6 \% \pm 7.2)$, and Metamonada $(1.9 \% \pm 1.5)$ with a total of $3.1 \% \pm 2.9$ unclassified reads (Fig. 1B and Supplementary Table S6). The most abundant orders previously reported as eukaryotic parasites detected were Chromadorea, Trichostomatia, and Trichomonadida (Fig. 1C). Chromadorea highest abundances were in October (94.4\%) and lowest in February (40.0\%). While Trichostomatia increased in February (53.6\%), and Trichomonadida in May (7.3\%). Overall, ASVs classified as Chromadorea showed high diversity, indicating a diverse nematode community (Supplementary Fig. S1).

To study fungal gut communities, we analyzed the ITS2 region. A total of $71 \% \pm 16.8$ of sequences were unclassified to Kingdom, thus demonstrating a lack of information from Malagasy fungal organisms in databases (Fig. 1D and Supplementary Table S7). When studying the gut mycobiome the separation between symbionts and environmental fungi using metagenomic approaches is challenging [24, 82]. Especially in redfronted lemurs, who feed on Fungi and plants, which potentially harbor fungal pathogens. Thus, we extracted only those fungal genera described before as gut symbionts [24]. We detected these genera in relative abundances <1\%: Cryptococcus, Agaricus, Candida, Saccharomyces, Malassezia, and Clavispora whereas other genera like Cladosporium, Aspergillus, Fusarium, and Penicillium were present in relative abundances $>1 \%$. In a phylogenetic analysis calculated from the 20 most abundant unclassified ASVs against ITS2 sequences from some of the fungal genera described as inhabitants of the gut mycobiome only one ASV was phylogenetically related to Cladosporium (Fig. 1E). Also, no similar sequences were detected in the NCBI database.

The archaeal community was assessed with $16 \mathrm{~S}$ rRNA gene analysis in a smaller set of samples using two different sets of primers aiming to recover sequences of different lineages. In both cases, only Methanomethylophilaceae was identified (Supplementary Tables 8 and 9). Thus, the archaeal community has a low diversity and comprises members also known from the gut of great apes and humans [83, 84].

\section{Temporal variations of the entire gut bacterial community composition}

The five most abundant genera comprise mostly novel organisms for which only classification at the family level was possible 
A

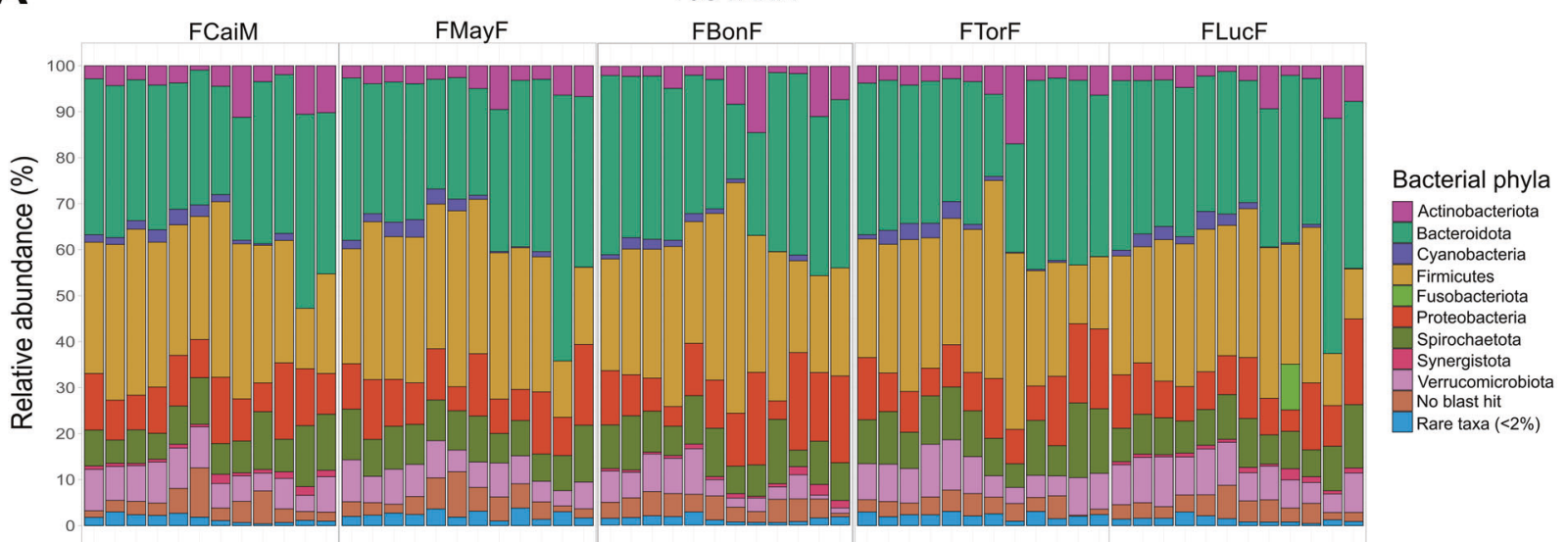

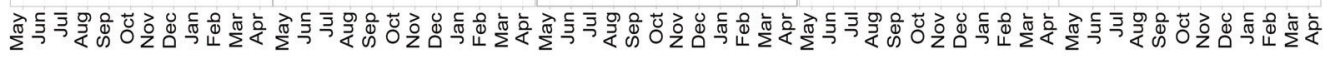

B

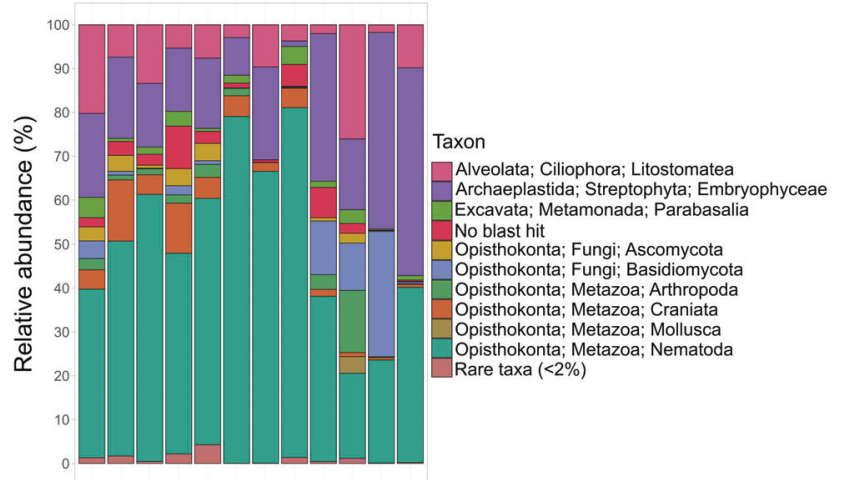

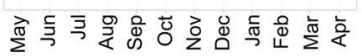

D

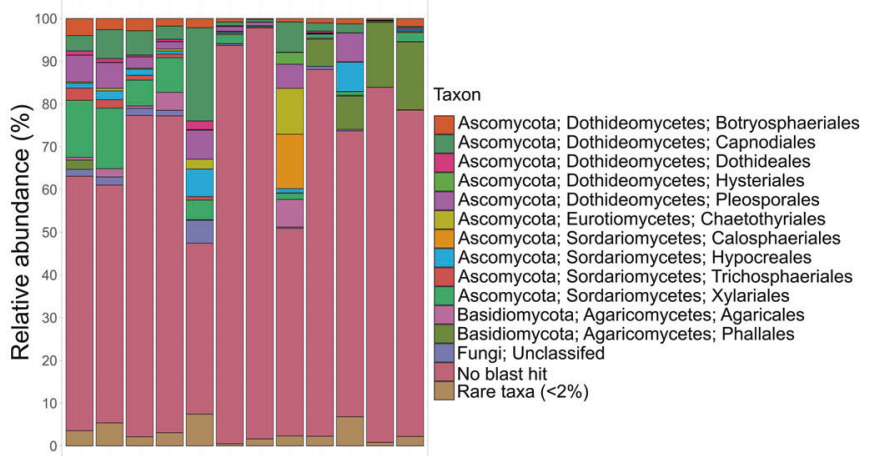

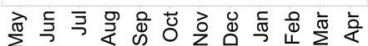

C

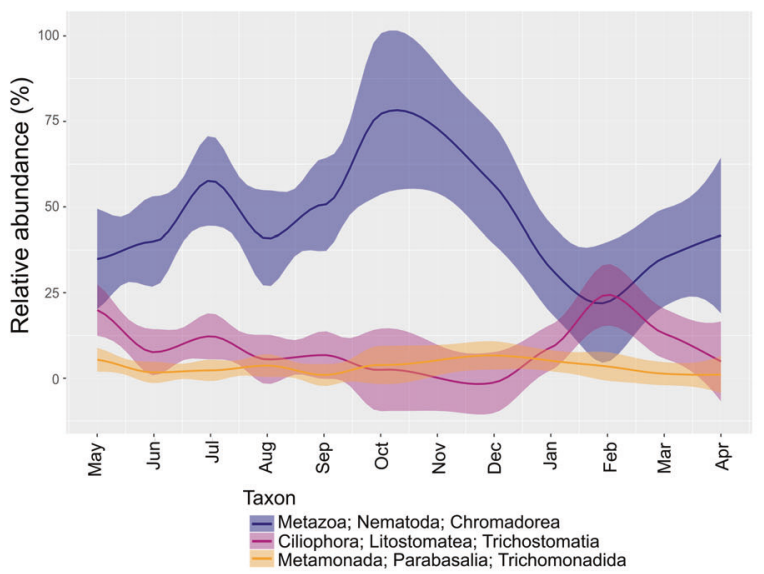

E

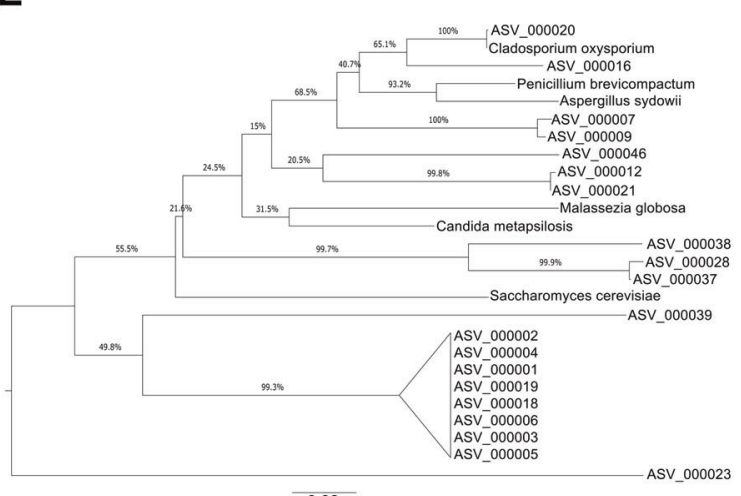

0.08

Fig. 1 Prokaryotic and eukaryotic communities from the gut of redfronted lemurs during the study period from May 2018 until April 2019. A Monthly relative abundances of bacterial phyla for the five studied individuals as determined from $16 \mathrm{~S}$ rRNA gene sequencing. Bar charts depict relative abundances of bacterial phyla from normalized counts for each individual per month. All phyla with abundances $<2 \%$ were grouped as rare taxa. B Eukaryotic organisms detected in the fecal samples through $18 \mathrm{~S}$ rRNA gene sequencing. Bar charts show monthly relative abundances of eukaryotic classes from normalized counts. All phyla with abundances $<2 \%$ were grouped as rare taxa. C Monthly fluctuations in the relative abundances of Chromadorea, Trichostomatia, and Trichomonadida. Linecharts depict relative abundances of normalized counts of the detected eukaryotic parasites or endosymbionts in the fecal samples. D Fungal organisms detected in the fecal samples through ITS2 sequencing. Barcharts display monthly relative abundances of fungal orders from normalized counts. All taxa with abundances $<2 \%$ were unified as rare taxa. E Maximum likelihood phylogenetic tree of the unclassified ITS2 ASVs against representative Fungi.

(Fig. 2A). These genera belong to the four families of Prevotellaceae $(14.6 \% \pm 7.4)$, Spirochaetaceae $(8.9 \% \pm 3.1)$, Rikenellaceae $(5.7 \% \pm$ 4.1) and Kiritimatiellae (5.1\% \pm 2.4$)$. The fifth most abundant genus was Sutterella $(3.9 \% \pm 2.3)$. All showed monthly fluctuations in their abundances, which were not always consistent among individuals
(Supplementary Fig. S2). The top 20 most abundant genera also presented monthly and individual differences in their relative abundances (Supplementary Fig. S3). A PERMANOVA test confirmed the $\beta$-dissimilarities were significantly different between individuals $(p<0.002)$. 
A
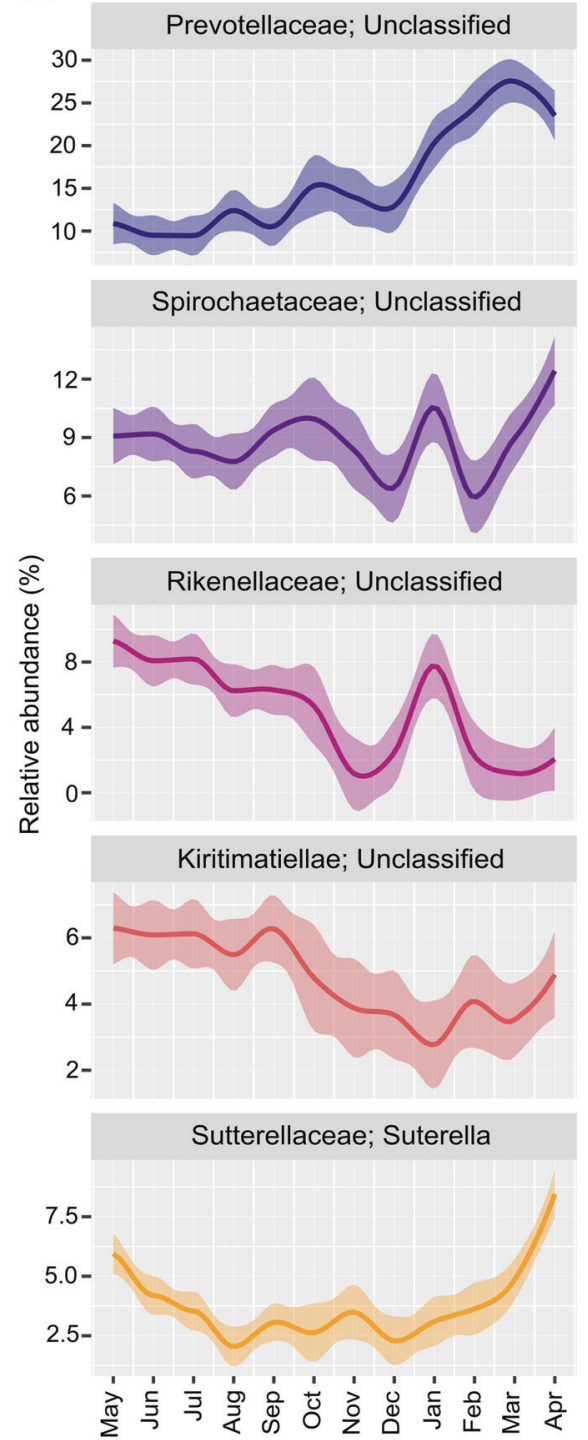

B
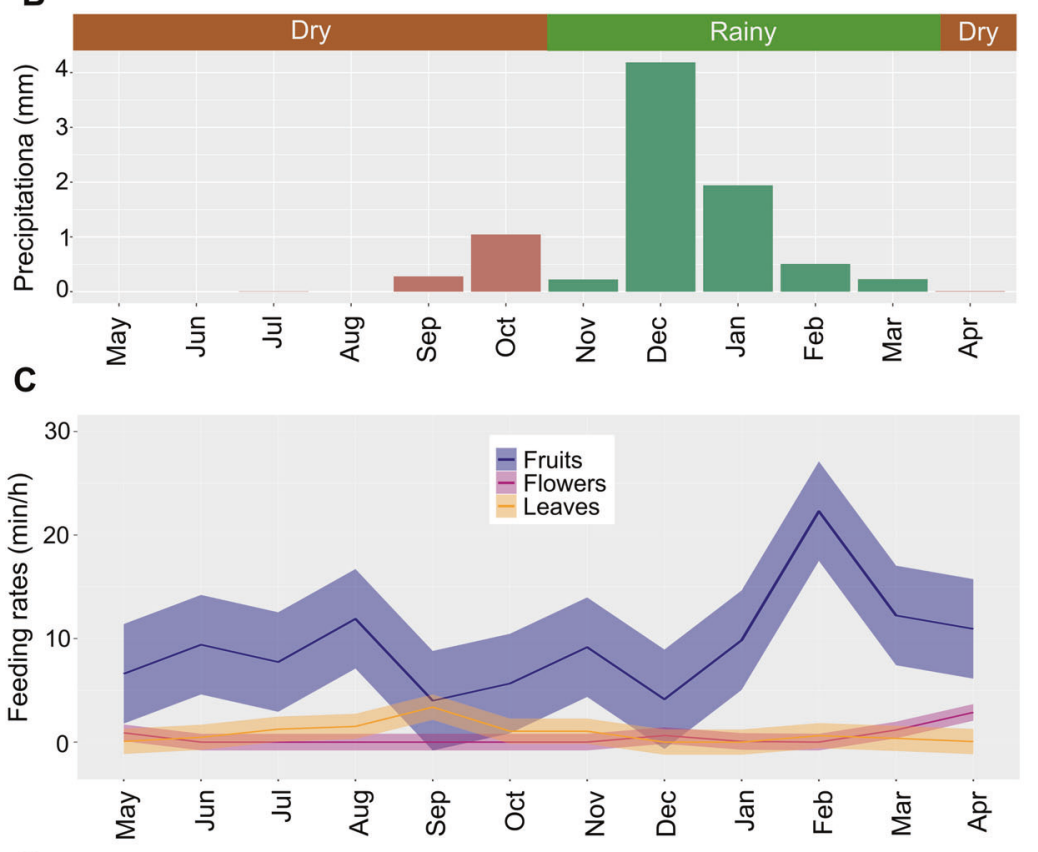

D

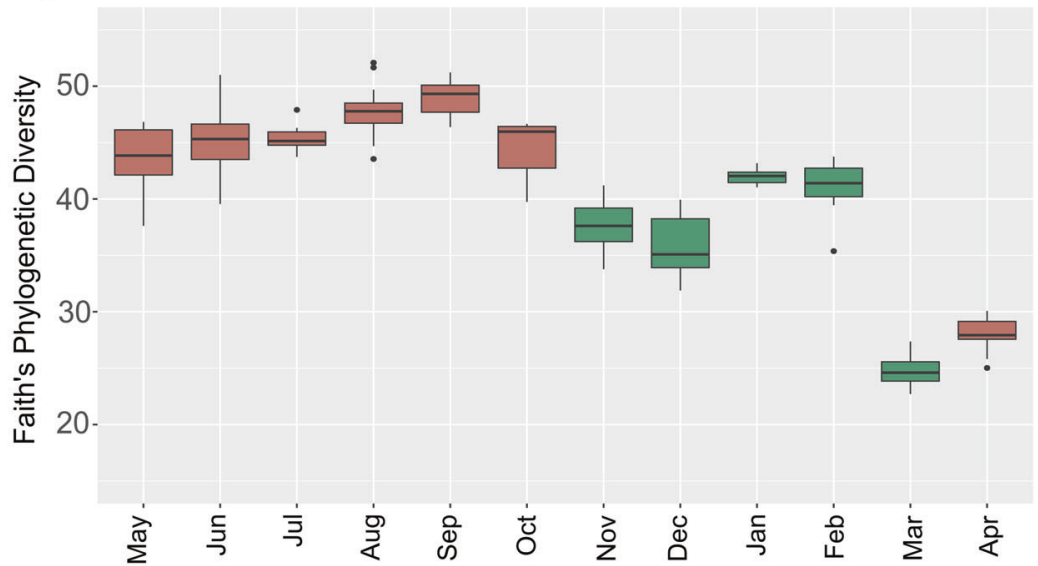

Fig. 2 Monthly fluctuations in most abundant bacterial genera and alpha diversity detected in fecal samples from redfronted lemurs from May 2018 to April 2019. A Top 5 most abundant bacterial genera and their monthly changes for all studied individuals. Line charts display relative abundances from normalized counts. B Mean monthly precipitation calculated from records of daily precipitation and seasons from the study period. C Monthly feeding rates on fruits, leaves, and flowers determined through behavioral focal observations. D Monthly variations in alpha diversity measured by Faith's Phylogenetic Diversity Index of all studied individuals.

Seasons were defined following previous publications [17], however, during our study rainfall increased at the end of the dry season (Fig. 2B), and feeding behaviors varied across months (Fig. 2C). Alpha diversity increased during the dry season with a maximum between August and October (Fig. 2D). The PD value fluctuated during the whole rainy season and was lower compared to the dry season. Monthly alpha diversity changes followed the same pattern in all individuals (Supplementary Fig. S4).

ANCOM analysis revealed that 75 genera showed significant differential abundance between dry and rainy seasons (Supplementary Fig. S5). We focused on taxa classified with relative abundances $\geq 1 \%$ (Fig. $3 A$ ).

Mean monthly precipitation, consumption of fruits, leaves, and/ or flowers and the rate of affiliative interactions correlated to the temporal variations in $\beta$-diversity. Samples from the dry season clustered together unlike the samples from the rainy season (Fig. 3B), and season $(p=0.001)$, precipitation $(p=0.001)$, feeding on fruits $(p=0.003)$, leaves $(p=0.044)$, flowers $(p=0.001)$, and affiliative interactions $(p=0.006)$.

The LMM detected taxon-specific effects (full-null model comparison; permutation test: $p=0.001)$ of flower $(p=0.001)$ and fruit feeding $(p=0.001)$, and affiliative interactions $(p=0.043)$ on the overall bacterial community composition (Supplementary Table S10) the following: exhibited significant correlations. We thus inspected the 20 taxa for which the taxon-specific effect deviated most from the average effect across all taxa for each significant predictor (Fig. 3C and Supplementary Fig. S6-9).

A time series analysis of WUnifrac distances against time between sample collection confirmed temporal variations on individual level (Supplementary Fig. S10 and Supplementary Table S11). Thus, the longer the timespan between the samples, the more dissimilar were the gut bacterial communities.

The LMM for the alpha diversity (full-null model comparison: $p=$ 0.003 ) detected an effect of feeding on leaves ( $p=0.055$, Fig. 4B) which correlated with an increase in PD, while the rates of flower 
A

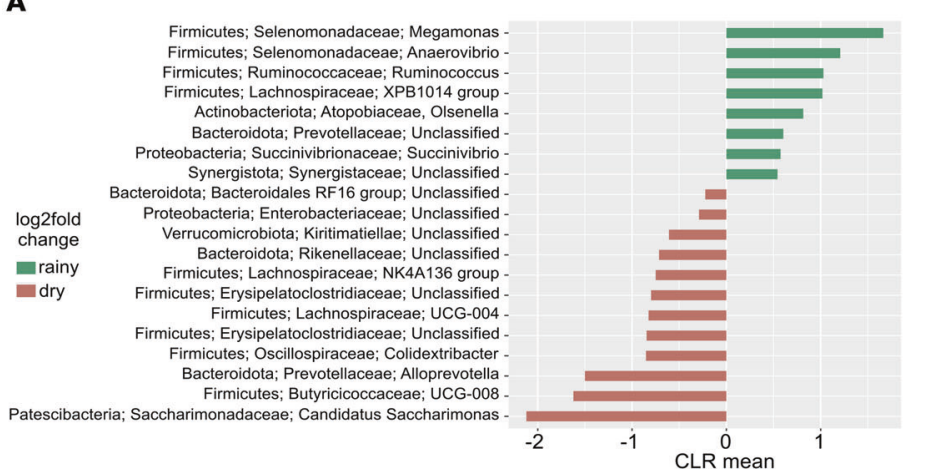

B

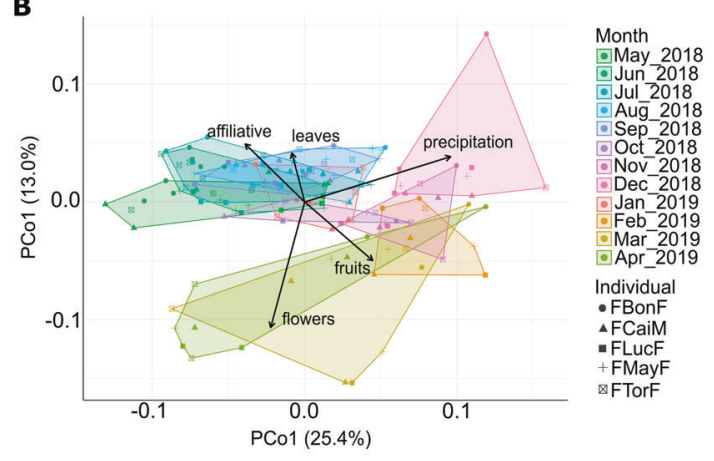

C

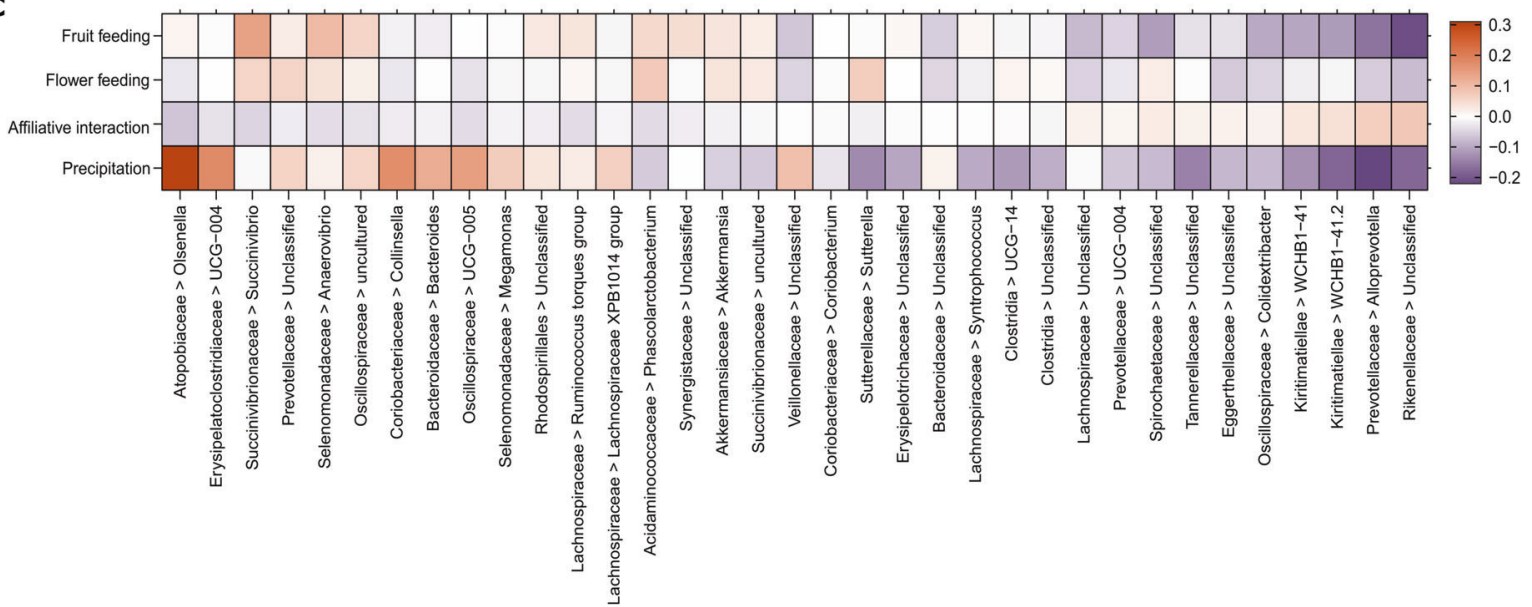

Fig. 3 Seasonal variations of bacterial genera, beta diversity, and composition of the entire gut bacterial community of redfronted lemurs from May 2018 until April 2019. A Log2f fold changes in the mean abundances of bacterial genera between dry and rainy season as detected with ANCOM 2.1. B PCOA based on weighted Unifrac of the bacterial community and environmental fit analysis depicting significant correlations between temporal fluctuations in beta diversity and the environmental, diet and social factors investigated. C Heatmap showing the 20 bacterial genera for which taxon-specific effects differed most from the average across all taxa as detected in a LMM estimating the effects of diet and affiliative interactions on community composition. The image displays the test predictors for which an effect was detected, feeding on flowers and fruits, and affiliation rates. Precipitation was included as the control predictor. Positive effects are depicted with orange, whereas negative effects are colored in purple.

A

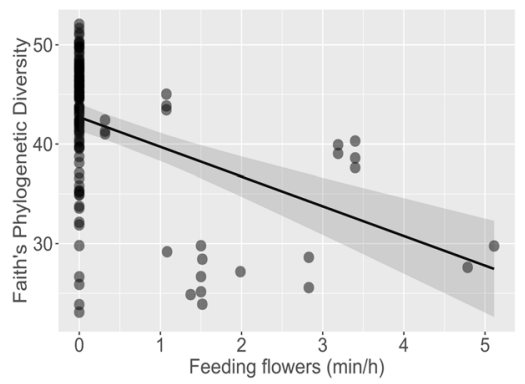

B

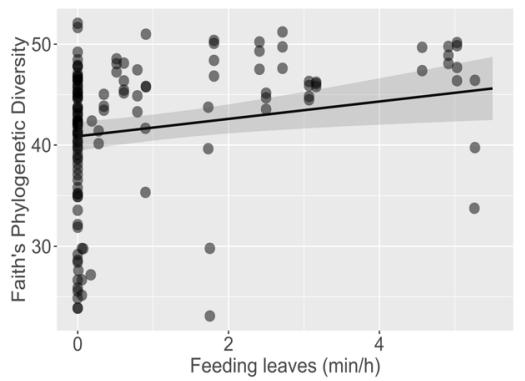

C

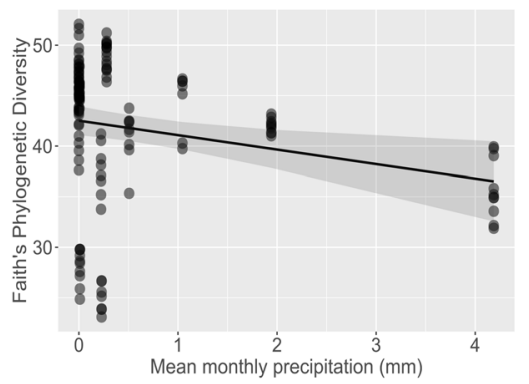

Fig. 4 Environmental and dietary factors driving the monthly fluctuations in alpha diversity of the entire bacterial community measured with the Faith's Phylogenetic Diversity index. A Monthly rates of flower consumption $(\mathrm{min} / \mathrm{h})$ correlate negatively with alpha diversity. B Monthly rates of leaves feeding $(\mathrm{min} / \mathrm{h})$ correlate positively with a higher alpha diversity. C Mean monthly precipitation correlates negatively with alpha diversity. The effects of diet, affiliation rates and precipitation were determined with a LMM.

consumption and mean monthly precipitation correlated negatively with PD (flowers: $p=0.002$; Fig. $4 \mathrm{~A}$; monthly rainfall: $p=0.039$, Fig. 4C, Supplementary Table S12). An effect of dietary changes on bacterial community composition was further confirmed by significant correlations from the plant diet deduced from the $18 \mathrm{~S}$ rRNA gene analysis (Supplementary Fig. S11A) to the fluctuations of the bacterial community (Supplementary Fig. S11B) $(p=0.001$; Supplementary Fig. S11C).

\section{Potential active bacterial community in the redfronted lemur gut}

The potentially active bacterial communities were analyzed from one sample per individual per month. The five most abundant phyla detected in the active community were Firmicutes $(56.1 \% \pm 13.1)$, Bacteroidota (16.5\% \pm 6.1$)$, Actinobacteriota $(9.9 \% \pm 4.4)$, Proteobacteria $(5.2 \% \pm 2.2)$, and Spirochaetota $(4.9 \% \pm 2.2)$ (Supplementary Fig. S12A). The five most abundant genera were Colidextribacter 
A
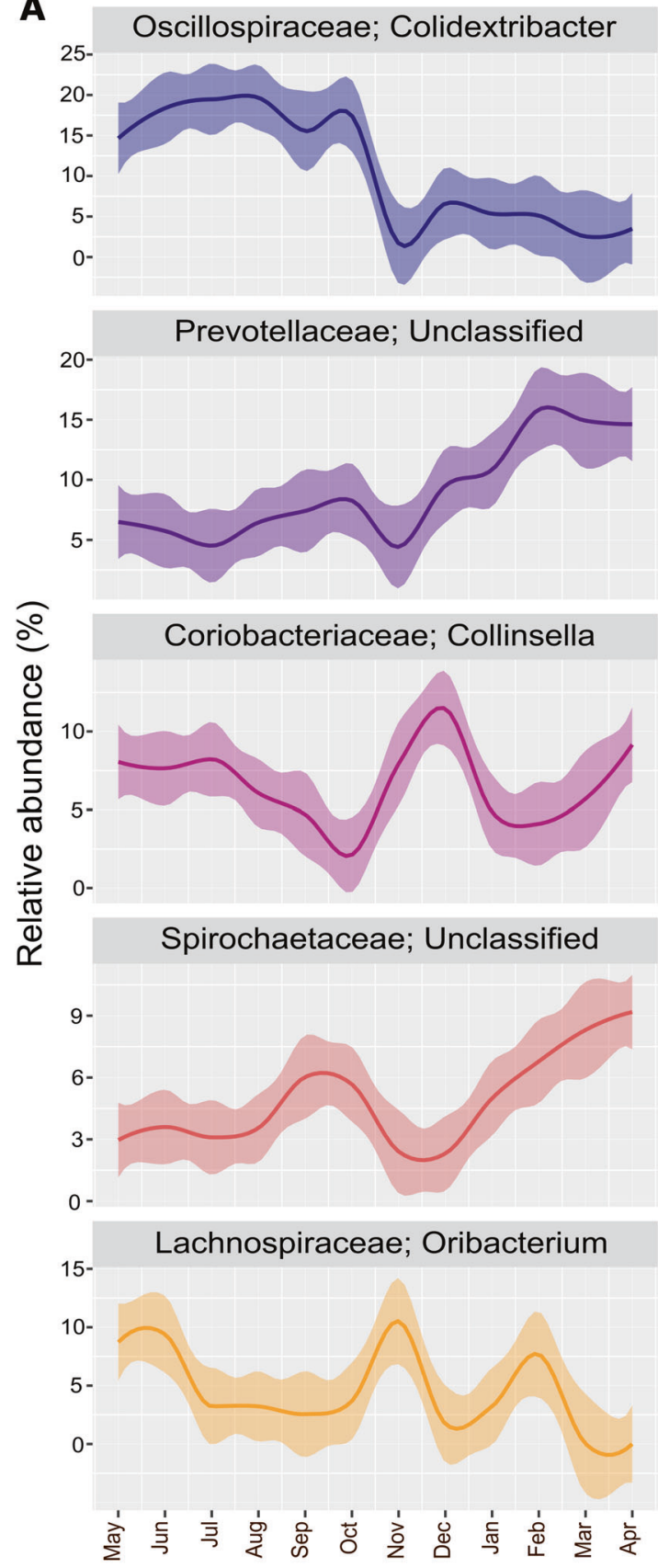

B
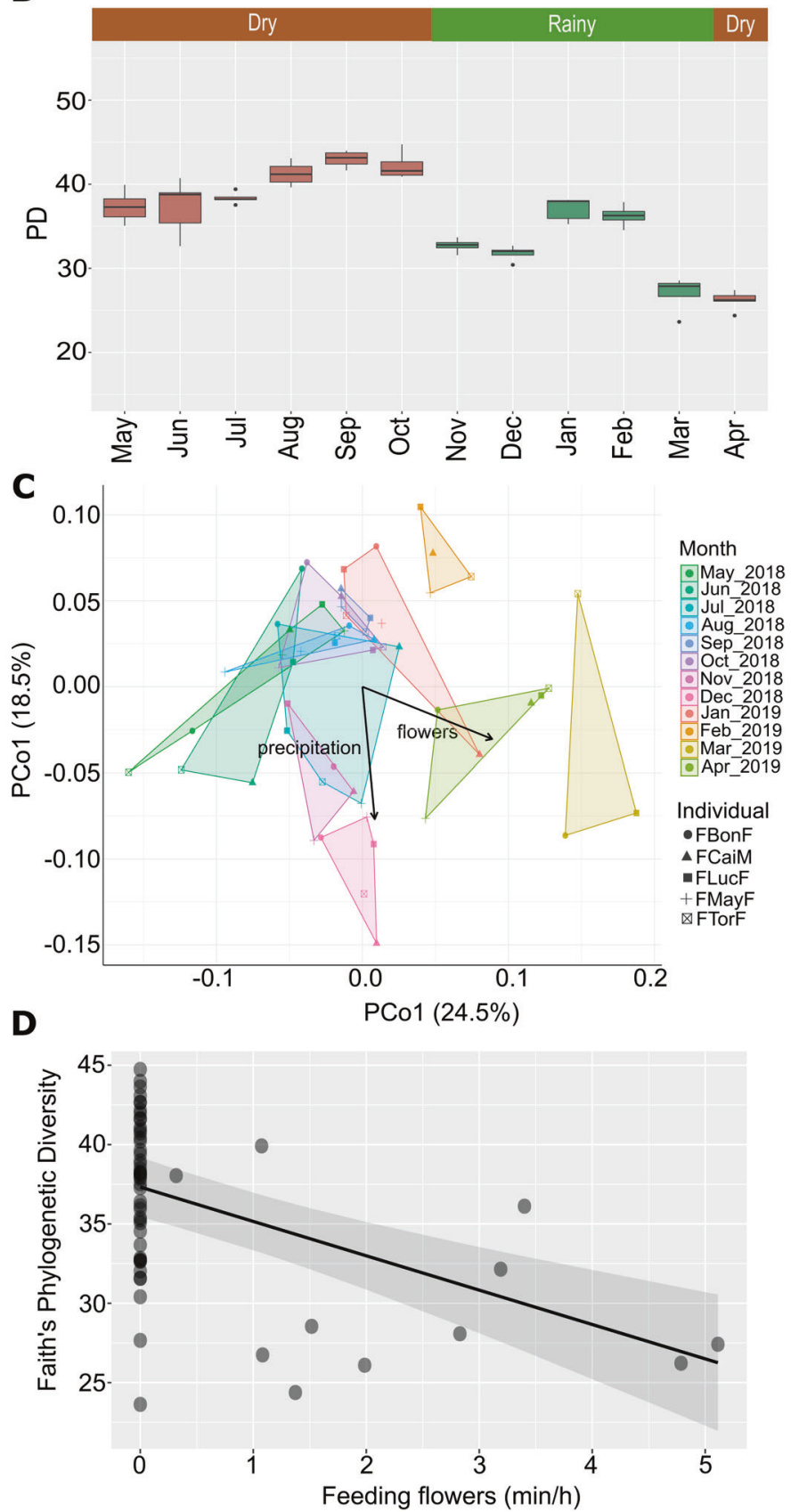

Fig. 5 Monthly fluctuations in the bacterial composition and alpha diversity of the active bacterial community in fecal samples from redfronted lemurs from May 2018 to April 2019. A Top five most abundant bacterial genera and their monthly changes for all studied individuals. Line charts display relative abundances from normalized counts. B Monthly variations in alpha diversity measured by Faith's Phylogenetic Diversity Index in all studied individuals. C PCoA from WUnifrac of the bacterial community and environmental fit analysis depicting significant correlations between temporal fluctuations in beta diversity and the environmental, diet and social factors investigated. D Monthly rates of flower consumption $(\mathrm{min} / \mathrm{h})$ correlate negatively with alpha diversity. The effects of diet, affiliation rates and precipitation on alpha diversity were determined with an LMM.

$(11.5 \% \pm 8.1)$, Prevotellaceae-Unclassified $(8.8 \% \pm 4.8)$, Collinsella $(6.7 \% \pm 3.4)$, Spirochaetaceae-Unclassified $(4.8 \% \pm 2.8)$, and Oribacterium $(4.5 \% \pm 4.6)$ (Fig. $5 \mathrm{~A}$ and Supplementary Fig. S12A). The top 20 most abundant genera were also investigated, which presented monthly and individual differences in their relative abundances (Supplementary Fig. S13).

The ANCOM analysis revealed that 40 genera exhibited significantly different relative abundances between seasons. Most exhibited abundances $<1 \%$ or were only classifiable to order level (Supplementary Fig. S14) leaving only Bacteroidales group RF16 with lower abundances in the rainy season, whereas Lachnospiraceae group XPB1014 and Fusobacterium had higher abundances in the rainy season. PD was higher during the dry season and more variable during the months of the rainy season, like at the entire community level (Fig. 5B). The PCoA did not show seasonal clustering (Fig. 5C). However, the environmental fit analysis detected correlations of season $(p=0.007)$, feeding on flowers $(p=0.003)$ and precipitation $(p=0.013)$ with the monthly alterations of the bacterial community (Fig. 5C). Mantel correlation tests of the $\beta$-dissimilarities and the timespan between sample 
collection for each individual were significant (Supplementary Table 13). Only feeding on flowers $(p=0.002)$ was associated with an effect in alpha diversity correlating with a decrease (Fig. 5D, Supplementary Table S14).

For comparison of entire with active communities a PCoA from WUnifrac with the reduced sample size was calculated also at entire community level. Precipitation $(p=0.003)$ and feeding on fruits $(p=0.056)$ and flowers $(p=0.002)$ were significantly correlated (Supplementary Fig. S15A). The comparison between the PCoAs from the entire and active community with the protest test from the Procrustes analysis detected significant correlations $(p=0.001)$. Thus, they were not significantly different (Supplementary Fig. S15B).

Functional predictions performed for the active community assigned $51.7 \%$ of the ASVs to an entry of the Faprotax database. Chemoheterotrophy $(21.6 \% \pm 3.6)$ and fermentation $(21.3 \pm 3.7)$ were the most abundant metabolisms, with a peak during the rainy season from October to January coinciding with an increase in fruit feeding (Fig. 2C and Supplementary Fig. S16).

\section{DISCUSSION}

Our longitudinal approach coupled with a dense sampling regime and behavioral data allowed us to detect in detail the temporal fluctuations of the gut microbial communities from redfronted lemurs. We determined the entire bacterial community changed accordingly to a higher consumption of fruits and flowers, and variations in affiliative interactions. Hence, the bacterial community quickly adapted to monthly changes in the diet but also to the host social behavior. Moreover, we characterized the potentially active bacterial community, which also underwent temporal fluctuations that correlated but only to flower consumption. The overall composition of the entire and the active bacterial communities were not significantly different, but the most abundant genera differed. The eukaryotic communities also presented temporal fluctuations and includes undescribed organisms.

\section{Unknown genera inhabit the gut microbiome of redfronted lemurs}

The most abundant bacterial phyla identified were Bacteroidota and Firmicutes similarly to other primates and humans $[12,85,86]$. Spirochaetota was also detected in high abundances, coinciding with previous reports from other primates and a cross-sectional study from the same species $[86,87]$. A previous study in the same lemur species detected only low abundances of treponemes but higher abundances of Cyanobacteria and Firmicutes [87]. However, in this study, samples were not placed in preservation solution for a time span of $12 \mathrm{~h}$, which might have altered the bacterial community [87].

The impossibility to classify the most abundant bacteria to taxonomic resolutions below family level highlights the presence of yet unclassified microorganisms in the gut of redfronted lemurs, as described in other non-human primates [86]. While the classifiable taxa are reported inhabitants of the gut from humans and other non-human primates [8, 11, 13, 86, 88]. Genera from Prevotellaceae and Spirochaetaceae, have been associated to plantbased diets providing pathways for their metabolization $[11,85,86]$. Rikenellaceae ferments carbohydrates and proteins [89]. Taxa from Verrucomicrobiota have been reported as mucinutilizers [11]. Less is known about the metabolic role of Sutterella, a common inhabitant of the human gut [90].

\section{The potential active bacterial community has a lower alpha diversity and differs in the most abundant taxa}

The potential active community had higher relative abundances of Firmicutes and a lower alpha diversity compared to the entire community. There are several possible explanations for the lower alpha diversity detected. First, redundancy of metabolisms in the bacterial community due to a pool of phylogenetically different community members capable to degrade the same substrates, which are not all active at the same time [2, 16, 91]. Second, community resilience, with other members in dormant stages that allow further functional adaptations when the environmental conditions change $[16,91]$. Third, differences in the copy numbers of $16 \mathrm{~S}$ rRNA genes between taxa, inflating the abundance of a taxa but not portraying the actual functional scenario [15, 16, 91]. However, the entire and active community are not significantly different and follow similar temporal fluctuations. Therefore, studying only the entire community provides insights into the temporal fluctuations of the gut microbiome, but studying the active community indicated functionally important active taxa can go unnoticed because of their lower abundances at entire community level. Regarding the most abundant genera detected differing from the entire community, there is no information about the metabolism of Colidextribacter, while Collinsella and Oribacterium are polysaccharide degraders coinciding with the lemurs' diet [92-94].

The functional predictions from the active community indicated an increase in fermentation and chemoheterotrophy during the rainy season possibly associated to the higher consumption of fruits and flowers $[8,9,12,14]$. However, we did not detect an augmentation of cellulolytic metabolism correlating with leaf consumption during the dry season. Since we performed metabolic predictions from taxonomy, we consider this is caused by the limited and biased metabolic information for certain taxa.

\section{Dietary changes have an effect in the temporal fluctuations of the gut microbiome}

The collection of behavioral data and the dietary assessment performed with the $18 \mathrm{~S}$ rRNA gene data allowed us to confirm temporal fluctuations of the gut microbiome correlate to dietary changes. We detected differentially abundant taxa for the rainy season, correlations of flower and fruit consumption to the temporal variations in $\beta$-diversity, and taxon-specific effects of flower and fruit consumption in bacterial composition. Flowers and fruits are high in non-structural polysaccharides like monoand disaccharides, but flowers contain more protein whereas fruits have a higher lipid content [95-97]. The positively affected taxa by the consumption of these plant parts coincide with these observations, since they are reported fermenters of mono- and disaccharides, like Succinivibrio, Oscillospiraceae, and Prevotellaceae, while Anaerovibrio, metabolizes glycolipids [85, 98-100]. Furthermore, Succinivibrio and Anaerovibrio produce succinate from their fermentations which in turn is the energy source of Phascolarctobacterium, another positively affected taxon $[98,99,101]$. The correlation of flower consumption with a lower alpha diversity suggests that a diverse gut microbial community is not needed for the digestion of flowers, coinciding with their less complex biochemical composition [96, 97].

Against our expectations, consumption of leaves only correlated to $\beta$-diversity changes from the dry season but did not influence the overall bacterial composition. However, leaf consumption correlated to higher alpha diversities, also reported in the Hadza community and baboons [11, 14]. As leaves have complex structural polysaccharides like hemicellulose, cellulose, and lignin, this indicates that a more diverse bacterial community is needed for the processing of the structural polysaccharides from a leaf diet [97].

Social interactions have an effect in the temporal fluctuations of the gut microbiome

Affiliative interactions correlated to the changes in $\beta$-diversity and influenced the overall gut microbiome composition. Lemurs use their toothcomb to groom themselves and others, by doing so, they can uptake microorganisms present on their furs and anogenital regions [102]. Rikenellaceae, Alloprevotella, Kiritimatiellae-WCHB1-41, and 
Spirochaetaceae were positively affected by affiliative interactions, indicating that they are transmitted via affiliative interactions. Social interactions correlated with $\beta$-diversity fluctuations in the dry season as well. During this period, social behaviors like mating, birth, and social thermoregulation to cope with the low temperatures occur, increasing microbe transmission $[19,103]$. Nonetheless, there were no births during our study period suggesting social thermoregulation played a more important role [19].

\section{Correlations between precipitation and temporal fluctuations of the gut microbiome}

Precipitation correlated with the fluctuations in $\beta$-diversity and a lower alpha diversity during the rainy season. Redfronted lemurs drink from waterholes and temporary ponds during the rainy season, whereas in the dry season, they drink from partially dry water holes in the river having higher microbial loads [20]. Higher precipitation resulting in water sources with lower microbial loads decreased alpha diversity and correlated to changes in $\beta$-diversity. Thus, taxa with higher abundances in the dry season could be ingested from drinking at the river waterholes like KiritimatiellaeWCHB1-41, which was impacted negatively by higher precipitation and has been previously isolated from environmental water suggesting transmission from water sources [104].

Gut of redfronted lemurs is inhabited by a great diversity of molecularly uncharacterized helminths and protozoa

The gut of all individuals harbored helminth and protozoan organisms over the entire year. These were classified only at the order level because they had high identity but low coverage to parasites of humans or livestock at higher taxonomical resolution. We detected a high prevalence of the Chromadorea and suspect most are from Lemuricola vauceli or Callistoura of Oxyuridae; however, genetical information from the V4 region of these organisms is absent in databases [18, 22]. This high prevalence has been previously detected morphologically but not in other metabarcoding studies [18, 22, 23]. Furthermore, our phylogenetic analysis detected other families like Trichuridae and Strongyloididae confirming a great diversity of nematodes inhabiting these lemurs [22].

The sequences detected from Trichostomatia possibly belong to Balantidium, following previous microscopical reports of this lemur species $[22,23]$. Moreover, the identified Trichomonadida, possibly a novel organism, was not detected before in microscopical studies, only in amplicon-based reports $[22,23]$. The differences in the taxa detected between this study and a previous metabarcoding report might be due to the amplification of different regions of the $18 \mathrm{~S}$ rRNA gene, we used the V4 while in other studies the V3V4 and V3-V5 were investigated [23, 31].

\section{The gut mycobiome of redfronted lemurs is comprised by novel fungi}

We detected in low relative abundances fungal genera described as human gut symbionts, suggesting the gut mycobiome of redfronted lemurs has low abundances and diversity, as reported in humans [24]. The majority of the ASVs were unclassifiable, even after performing a phylogenetic analysis of the most abundant sequences against representative fungi, confirming the deficiency in genomic information from fungal organisms found in Madagascar and the gut of wild-living animals [24, 82]. The observed variation of the unclassified taxa between months could portray changes in the gut mycobiome. Nonetheless, it should be considered that some of the detected taxa might derive from diet, as redfronted lemurs fed on fungi or fungal plant pathogens $[25,82]$.

\section{CONCLUSION}

Fruit and flower consumption, affiliative interactions and water sources are important drivers of the temporal fluctuations of the gut bacterial communities from redfronted lemurs. Thus, displaying how this bacterial community adapts to the host diet and behavior following temporal changes. Eukaryotic gut communities also fluctuate monthly and are very diverse. Our results affirm intricate host-microbiome interactions in the gut of redfronted lemurs are affected by the host diet, precipitation, and social behavior. To our knowledge, this is the first 1-year study combining thorough sampling and individual behavioral data collection allowing the detection of direct links between temporal fluctuations of bacterial taxa and consumption of specific food items and social behavior. Longitudinal studies as the one performed here capture better the effects of seasonality on the fluctuations of the gut microbiome, diet, and social behaviors than cross-sectional approaches.

\section{REFERENCES}

1. McKenney EA, Koelle K, Dunn RR, Yoder AD. The ecosystem services of animal microbiomes. Mol Ecol. 2018;27:2164-72.

2. Miller ET, Svanbäck R, Bohannan BJM. Microbiomes as metacommunities: understanding host-associated microbes through metacommunity ecology. Trends Ecol Evol. 2018;33:926-35.

3. Johnson AJ, Vangay P, Al-Ghalith GA, Hillmann BM, Ward TL, Shields-Cutler RR, et al. Daily sampling reveals personalized diet-microbiome associations in humans. Cell Host Microbe. 2019;25:789-802.

4. Björk JR, Dasari M, Grieneisen L, Archie EA. Primate microbiomes over time: Longitudinal answers to standing questions in microbiome research. Am J Primatol. 2019;81:1-23.

5. Sun B, Wang X, Bernstein S, Huffman MA, Xia DP, Gu Z, et al. Marked variation between winter and spring gut microbiota in free-ranging Tibetan Macaques (Macaca thibetana). Sci Rep. 2016;6:1-8.

6. Wu Q, Wang $X$, Ding $Y$, Hu $Y$, Nie $Y$, Wei $W$, et al. Seasonal variation in nutrient utilization shapes gut microbiome structure and function in wild giant pandas. Proc R Soc B Biol Sci. 2017;284:20170955.

7. Maurice $C F$, Knowles SCL, Ladau J, Pollard KS, Fenton A, Pedersen AB, et al. Marked seasonal variation in the wild mouse gut microbiota. ISME J. 2015;9: 2423-34.

8. Amato KR, Leigh SR, Kent A, Mackie Rl, Yeoman CJ, Stumpf RM, et al. The gut microbiota appears to compensate for seasonal diet variation in the wild black howler monkey (Alouatta pigra). Microb Ecol. 2014;69:434-43.

9. Orkin JD, Campos FA, Myers MS, Cheves Hernandez SE, Guadamuz A, Melin AD. Seasonality of the gut microbiota of free-ranging white-faced capuchins in a tropical dry forest. ISME J. 2019;13:183-96. 22.

10. Springer A, Fichtel C, Al-Ghalith GA, Koch F, Amato KR, Clayton JB, et al. Patterns of seasonality and group membership characterize the gut microbiota in a longitudinal study of wild Verreaux's sifakas (Propithecus verreauxi). Ecol Evol. 2017;7:5732-45.

11. Jagsi R, Jiang J, Momoh AO, Alderman A, Giordano SH, Buchholz TA, et al. Seasonal cycling in the gut microbiome of the Hadza Hunter-Gatherers of Tanzania. Science. 2017;357:802-6.

12. Hicks AL, Lee KJ, Couto-Rodriguez M, Patel J, Sinha R, Guo C, et al. Gut microbiomes of wild great apes fluctuate seasonally in response to diet. Nat Commun. 2018;9:1786.

13. Baniel A, Amato KR, Beehner JC, Bergman TJ, Mercer A, Perlman RF, et al. Seasonal shifts in the gut microbiome indicate plastic responses to diet in wild geladas. Microbiome. 2021;9:1-20.

14. Ren T, Grieneisen LE, Alberts SC, Archie EA, Wu M. Development, diet and dynamism: longitudinal and cross-sectional predictors of gut microbial communities in wild baboons. Environ Microbiol. 2016;18:1312-25.

15. Větrovský $T$, Baldrian P. The variability of the $16 \mathrm{~S}$ rRNA gene in bacterial genomes and its consequences for bacterial community analyses. PLoS One. 2013;8: e57923.

16. De Vrieze J, Pinto AJ, Sloan WT, ljaz UZ. The active microbial community more accurately reflects the anaerobic digestion process: 16S rRNA (gene) sequencing as a predictive tool. Microbiome. 2018;6:63.

17. Kappeler PM, Fichtel C. A 15-year perspective on the social organization and life history of sifaka in Kirindy Forest. In: Long-term field studies of primates. Springer Berlin Heidelberg; 2012. p. 101-21.

18. Peckre LR, Defolie C, Kappeler PM, Fichtel C. Potential self-medication using millipede secretions in red-fronted lemurs: combining anointment and ingestion for a joint action against gastrointestinal parasites? Primates. 2018; 59:483-94.

19. Ostner J. Social thermoregulation in redfronted lemurs (Eulemur fulvus rufus). Folia Primatol. 2002;73:175-80. 
20. Amoroso CR, Kappeler PM, Fichtel C, Nunn CL. Water availability impacts habitat use by red-fronted lemurs (Eulemur rufifrons): An experimental and observational study. Int J Primatol. 2020;41:61-80.

21. Koch F, Ganzhorn JU, Rothman JM, Chapman CA, Fichtel C. Sex and seasonal differences in diet and nutrient intake in Verreaux's sifakas (Propithecus verreauxi). Am J Primatol. 2017;79:1-10.

22. Clough D. Gastro-intestinal parasites of red-fronted lemurs in Kirindy Forest, western Madagascar. J Parasitol. 2010;96:245-51.

23. Gogarten JF, Calvignac-Spencer S, Nunn CL, Ulrich M, Saiepour N, Nielsen HV, et al. Metabarcoding of eukaryotic parasite communities describes diverse parasite assemblages spanning the primate phylogeny. Mol Ecol Resour. 2020;20:204-15.

24. Lai GC, Tan TG, Pavelka N. The mammalian mycobiome: A complex system in a dynamic relationship with the host. Wiley Interdiscip Rev Syst Biol Med. 2019;11:e1438.

25. Mann AE, Mazel F, Lemay MA, Morien E, Billy V, Kowalewski M, et al. Biodiversity of protists and nematodes in the wild nonhuman primate gut. ISME J. 2020;14:609-22.

26. Grieneisen LE, Livermore J, Alberts S, Tung J, Archie EA. Group living and male dispersal predict the core gut microbiome in wild baboons. Integr Comp Biol. 2017;57:770-85.

27. Klindworth A, Pruesse E, Schweer T, Peplies J, Quast C, Horn M, et al. Evaluation of general 16S ribosomal RNA gene PCR primers for classical and nextgeneration sequencing-based diversity studies. Nucleic Acids Res. 2013;41:1-11.

28. Toju H, Tanabe AS, Yamamoto S, Sato H. High-coverage ITS primers for the DNAbased identification of ascomycetes and basidiomycetes in environmental samples. PLoS One. 2012;7:e40863.

29. Porat I, Vishnivetskaya TA, Mosher JJ, Brandt CC, Yang ZK, Brooks SC, et al. Characterization of archaeal community in contaminated and uncontaminated surface stream sediments. Microb Ecol. 2010;60:784-95.

30. Gantner S, Andersson AF, Alonso-Sáez L, Bertilsson S. Novel primers for $16 \mathrm{~S}$ rRNA-based archaeal community analyses in environmental samples. J Microbiol Methods. 2011;84:12-8.

31. Stoeck T, Bass D, Nebel M, Christen R, Jones MDM, Breiner H-W, et al. Multiple marker parallel tag environmental DNA sequencing reveals a highly complex eukaryotic community in marine anoxic water. Mol Ecol. 2010;19:21-31.

32. Bahram M, Anslan S, Hildebrand F, Bork P, Tedersoo L. Newly designed 16 S rRNA metabarcoding primers amplify diverse and novel archaeal taxa from the environment. Environ Microbiol Rep. 2019;11:487-94.

33. Berkelmann D, Schneider D, Hennings N, Meryandini A, Daniel R. Soil bacterial community structures in relation to different oil palm management practices. Sci Data. 2020;7:1-7.

34. Chen S, Zhou Y, Chen Y, Gu J. Fastp: An ultra-fast all-in-one FASTQ preprocessor. Bioinformatics. 2018;34:i884-90.

35. Zhang J, Kobert K, Flouri T, Stamatakis A. PEAR: A fast and accurate Illumina paired-end read mergeR. Bioinformatics. 2014;30:614-20.

36. Martin M. Cutadapt removes adapter sequences from high-throughput sequencing reads. EMBnet J. 2011;17:10-2.

37. Rognes T, Flouri T, Nichols B, Quince C, Mahé F. VSEARCH: A versatile open source tool for metagenomics. PeerJ. 2016;2016:1-22.

38. Edgar RC UNOISE2: improved error-correction for Illumina $16 \mathrm{~S}$ and ITS amplicon sequencing. bioRxiv. 2016;081257.

39. Edgar RC UCHIME2: improved chimera prediction for amplicon sequencing. bioRxiv. 2016;074252.

40. Altschul SF, Gish W, Miller W, Myers EW, Lipman DJ. Basic local alignment search tool. J Mol Biol. 1990;215:403-10.

41. Quast C, Pruesse E, Yilmaz P, Gerken J, Schweer T, Yarza P, et al. The SILVA ribosomal RNA gene database project: Improved data processing and webbased tools. Nucleic Acids Res. 2013;41:590-6.

42. Guillou L, Bachar D, Audic S, Bass D, Berney C, Bittner L, et al. The protist ribosomal reference database (PR2): A catalog of unicellular eukaryote small sub-unit rRNA sequences with curated taxonomy. Nucleic Acids Res. 2013;41:597-604.

43. Nilsson RH, Larsson KH, Taylor AFS, Bengtsson-Palme J, Jeppesen TS, Schigel D, et al. The UNITE database for molecular identification of fungi: Handling dark taxa and parallel taxonomic classifications. Nucleic Acids Res. 2019;47:D259-64.

44. Yarza P, Yilmaz P, Pruesse E, Glöckner FO, Ludwig W, Schleifer KH, et al. Uniting the classification of cultured and uncultured bacteria and archaea using $16 \mathrm{~S}$ rRNA gene sequences. Nat Rev Microbiol. 2014;12:635-45.

45. Louca S, Parfrey LW, Doebeli M. Decoupling function and taxonomy in the global ocean microbiome. Science. 2016;353:1272-7.

46. Team Rc. R: a language and environment for statistical computing. Vienna, Austria: R Foundation for Statistical Computing; 2020. Available from: https:// www.r-project.org.

47. Team Rs. RStudio: Integrated Development for R. Boston, MA: RStudio; 2019.

48. Andersen KS, Kirkegaard RH, Albertsen M ampvis2: an R package to analyse and visualise 16S rRNA amplicon data. bioRxiv. 2018;10-1.
49. Paradis E, Claude J, Strimmer K. APE: Analyses of phylogenetics and evolution in R language. Bioinformatics. 2004;20:289-90.

50. Wickham H, Rstudio. stringr: Simple, consistent wrappers for common string operations. 2019. Available from: https://cran.r-project.org/package=stringr.

51. Wickham H. Reshaping data with the reshape package. J Stat Softw. 2007;21:1-20.

52. Dowle M, Srinivasan A, Gorecki J, Chirico M, Stetsenko $P$, Short T, et al. data. table: Extension of "data.frame". 2019. Available from: https://cran.r-project.org/ package $=$ data.table.

53. Wickham H, Averick M, Bryan J, Chang W, McGowan L, François R, et al. Welcome to the Tidyverse. J Open Source Softw. 2019;4:1686.

54. Wickham H ggplot2: Elegant graphics for data analysis. New York: SpingerVerlag New York; 2016.

55. Chen L, Reeve J, Zhang L, Huang S, Wang X, Chen J. GMPR: A robust normalization method for zero-inflated count data with application to microbiome sequencing data. PeerJ. 2018;2018:1-20.

56. Katoh K, Standley DM. MAFFT multiple sequence alignment software version 7: Improvements in performance and usability. Mol Biol Evol. 2013;30:772-80.

57. Price MN, Dehal PS, Arkin AP. FastTree 2-Approximately maximum-likelihood trees for large alignments. PLoS One. 2010;5:e9490.

58. Rambaut A. FigTree-tree figure drawing tool. Institute of Evolutionary Biology, University of Edinburg; 2020. Available from: http://tree.bio.ed.ac.uk/software/figtree.

59. Kembel SW, Cowan PD, Helmus MR, Cornwell WK, Morlon H, Ackerly DD, et al. Picante: $\mathrm{R}$ tools for integrating phylogenies and ecology. Bioinformatics. 2010;26:1463-4.

60. Marzano V, Mancinelli L, Bracaglia G, Del Chierico F, Vernocchi P, Di Girolamo F, et al. "Omic" investigations of protozoa and worms for a deeper understanding of the human gut "parasitome.". PLoS Negl Trop Dis. 2017;11:e0005916.

61. Mandal S, Van Treuren W, White RA, Eggesbø M, Knight R, Peddada SD. Analysis of composition of microbiomes: a novel method for studying microbial composition. Microb Ecol Heal Dis. 2015;26:27663.

62. Hothorn T, Hornik K exactRankTests: exact distributions for rank and permutation tests. 2019. Available from: https://cran.r-project.org/package=exactRankTests.

63. Pinheiro J, Bates D, DebRoy S, Sarkar D, Core Team R nlme: Linear and nonlinear mixed effects models. 2020. Available from: https://cran.r-project.org/ package $=$ nlme.

64. van den Boogaart $G$, Tolosana $R$ compositions: an $R$ package for compositional data analysis. 2020. Available from: http://www.stat.boogaart.de/compositions/.

65. Wickham H, Francois R, Core Team R, Rstudio, Jylänki J, Jorgensen M readr: Read rectangular text data. 2018. Available from: http://readr.tidyverse.org.

66. Oksanen J, Blanchet G, Friendly M, Kindt R, Legendre P, McGlinn D, et al. vegan: Community ecology package. 2019. Available from: https://cran.r-project.org/ package $=$ vegan .

67. Lozupone C, Lladser ME, Knights D, Stombaugh J, Knight R. UniFrac: An effective distance metric for microbial community comparison. ISME J. 2011:5:169-72.

68. Bates D, Mächler M, Bolker BM, Walker SC. Fitting linear mixed-effects models using Ime4. J Stat Softw. 2015;67:1-48.

69. Reitmeier S, Hitch TCA, Treichel N, Fikas N, Hausmann B, Ramer-Tait AE, et al. Handling of spurious sequences affects the outcome of high-throughput $16 \mathrm{~S}$ rRNA gene amplicon profiling. ISME COMMUN. 2021;1:31.

70. Sweeny AR, Lemon H, Ibrahim A, Nussey DH, Free A, McNally L. A mixed model approach for estimating drivers of microbiota community composition and differential taxonomic abundance (preprint). bioRxiv. 2020; https://doi.org/ 10.1101/2020.11.24.395715.

71. Barr DJ, Levy R, Scheepers C, Tily HJ. Random effects structure for confirmatory hypothesis testing: Keep it maximal. J Mem Lang. 2013;68:255-78.

72. Matuschek H, Kliegl R, Vasishth S, Baayen H, Bates D. Balancing type I error and power in linear mixed models. J Mem Lang. 2017;94:305-15.

73. Fox J, Weisberg S. An R companion to applied regression. 3rd ed. Thousand Oaks, California: Sage Publishing; 2019.

74. Bolker BM. Ecological models and data in R. New Jersey, USA: Princeton University Press; 2008.

75. Adams DC, Anthony $C D$. Using randomization techniques to analyse behavioural data. Anim Behav. 1996;51:733-8.

76. Baayen RH. Analyzing linguistic data. Cambridge: Cambridge University Press; 2008.

77. Bartón K MuMln: Multi-Model Inference. 2020. Available from: https://cran.rproject.org/package $=$ MuMIn.

78. Lüdecke D. sjPlot: Data Visualization for Statistics in Social Science. 2021. Available from: https://cran.r-project.org/package=sjPlot.

79. Edgar RC. MUSCLE: Multiple sequence alignment with high accuracy and high throughput. Nucleic Acids Res. 2004;32:1792-7.

80. Kumar S, Stecher G, Li M, Knyaz C, Tamura K. MEGA X: Molecular evolutionary genetics analysis across computing platforms. Mol Biol Evol. 2018;35:1547-9.

81. NCBI Resource Coordinators. Database resources of the National Center for Biotechnology Information. Nucleic Acids Res. 2018;46:D8-13. 
82. Nilsson RH, Anslan S, Bahram M, Wurzbacher C, Baldrian P, Tedersoo L. Mycobiome diversity: high-throughput sequencing and identification of fungi. Nat Rev Microbiol. 2019;17:95-109. 15.

83. Koskinen K, Pausan MR, Perras AK, Beck M, Bang C, Mora M, et al. First insights into the diverse human archaeome: specific detection of archaea in the gastrointestinal tract, lung, and nose and on skin. MBio. 2017;8:1-17.

84. Raymann K, Moeller AH, Goodman AL, Ochman H. Unexplored archaeal diversity in the great ape gut microbiome. mSphere. 2017;2:1-12.

85. Ley RE. Prevotella in the gut: choose carefully. Nat Rev Gastroenterol Hepatol. 2016;13:69-70.

86. Manara S, Asnicar F, Beghini F, Bazzani D, Cumbo F, Zolfo M, et al. Microbial genomes from non-human primate gut metagenomes expand the primate-associated bacterial tree of life with over 1000 novel species. Genome Biol. 2019;20:299.

87. Umanets A, de Winter I, IJdema F, Ramiro-Garcia J, van Hooft P, Heitkönig IMA, et al. Occupancy strongly influences faecal microbial composition of wild lemurs. FEMS Microbiol Ecol. 2018;94:1-13.

88. Greene LK, Clayton JB, Rothman RS, Semel BP, Semel MA, Gillespie TR, et al. Local habitat, not phylogenetic relatedness, predicts gut microbiota better within folivorous than frugivorous lemur lineages. Biol Lett. 2019;15:5-11.

89. Daniel H, Gholami AM, Berry D, Desmarchelier C, Hahne H, Loh G, et al. High-fat diet alters gut microbiota physiology in mice. ISME J. 2014;8:295-308.

90. Hiippala K, Kainulainen V, Kalliomäki M, Arkkila P, Satokari R. Mucosal prevalence and interactions with the epithelium indicate commensalism of Sutterella spp. Front Microbiol. 2016;7:1-13.

91. Heintz-Buschart A, Wilmes P. Human gut microbiome: Function matters. Trends Microbiol. 2018;26:563-74.

92. Ricaboni D, Mailhe M, Cadoret F, Vitton V, Fournier PE, Raoult D. 'Colidextribacter massiliensis' gen. nov., sp. nov., isolated from human right colon. New Microbes New Infect. 2017;17:27-9.

93. Qin P, Zou Y, Dai Y, Luo G, Zhang X, Xiao L. Characterization a novel butyric acidproducing bacterium Collinsella aerofaciens subsp. shenzhenensis subsp. nov. Microorganisms. 2019;7:78

94. Sizova MV, Muller PA, Stancyk D, Panikov NS, Mandalakis M, Hazen A, et al. Oribacterium parvum sp. nov. and Oribacterium asaccharolyticum sp. nov., obligately anaerobic bacteria from the human oral cavity, and emended description of the genus Oribacterium. Int J Syst Evol Microbiol. 2014;64:2642-9.

95. Amato KR, Garber PA. Nutrition and foraging strategies of the black howler monkey (Alouatta pigra) in Palenque National Park, Mexico. Am J Primatol. 2014;76:774-87.

96. White TCR. The significance of unripe seeds and animal tissues in the protein nutrition of herbivores. Biol Rev. 2011;86:217-24.

97. Ortmann S, Bradley BJ, Stolter C, Ganzhorn JU. Estimating the quality and composition of wild animal diets-a critical survey of methods. In: Hohmann G, Robbins $M$, Boesch $C$, editors. Feeding ecology in apes and other primates ecological, physical, and behavioral aspects. Cambridge: Cambridge University Press; 2006. p. 395-418.

98. Hippe H, Hagelstein A, Kramer I, Swiderski J, Stackebrandt E. Phylogenetic analysis of Formivibrio citricus, Propionivibrio dicarboxylicus, Anaerobiospirillum thomasii, Succinirnonas amylolytica and Succinivibrio dextrinosolvens and proposal of Succinivibrionaceae fam. nov. Int J Syst Evol Microbiol. 1999;49:779-82.

99. Privé F, Kaderbhai NN, Girdwood S, Worgan HJ, Pinloche E, Scollan ND, et al. Identification and characterization of three novel lipases belonging to families II and V from Anaerovibrio lipolyticus 5ST. PLoS One. 2013;8:e69076.

100. Flaiz M, Baur T, Brahner S, Poehlein A, Daniel R, Bengelsdorf FR. Caproicibacter fermentans gen. nov., sp. nov., a new caproate-producing bacterium and emended description of the genus Caproiciproducens. Int J Syst Evol Microbiol. 2020;70:4269-79.

101. Watanabe Y, Nagai F, Morotomi M. Characterization of Phascolarctobacterium succinatutens sp. Nov., an asaccharolytic, succinate-utilizing bacterium isolated from human feces. Appl Environ Microbiol. 2012;78:511-8.

102. Clough D, Heistermann M, Kappeler PM. Host intrinsic determinants and potential consequences of parasite infection in free-ranging red-fronted lemurs (Eulemur fulvus rufus). Am J Phys Anthropol. 2010;142:441-52.

103. Sarkar A, Harty S, Johnson KVA, Moeller AH, Archie EA, Schell LD, et al. Microbial transmission in animal social networks and the social microbiome. Nat Ecol Evol. 2020;4:1020-35.
104. van Vliet DM, Lin Y, Bale NJ, Koenen M, Villanueva L, Stams AJM, et al. Pontiella desulfatans gen. nov., sp. nov., and Pontiella sulfatireligans sp. nov., two marine anaerobes of the Pontiellaceae fam. nov. producing sulfated glycosaminoglycanlike exopolymers. Microorganisms. 2020;8:920. 18.

\section{ACKNOWLEDGEMENTS}

This project was funded by the Deutsche Forschungsgemeinschaft (FI 929/7-2, DA 374/13-2) and is embedded in the research unit "Sociality and Health in Primates" (SoHaPi). Research permits were granted by the Malagasy Ministère de I'Environnement et des Eaux et Forêts, the University of Antananarivo, and the Centre National de Formation, d'Etudes et de Recherche en Environnement et Foresterie. For support in sample and data collection, we thank Jean-Pierre Tolojanahary, Mamy Razafindrasamba, Tianasoa Andrianjanahary, Judit Stolla, Luise Waldow, and Alexandra Michiels. We thank Dr. Anja Poehlein, Melanie Heinemann, and Sarah Schüßler from the Göttingen Genomics Laboratory for amplicon sequencing. For discussion and statistical advice, we thank Dr. Roger Mundry and Dr. Louise Peckre.

\section{AUTHOR CONTRIBUTIONS}

CF and RD obtained the funding and designed the study. TM conducted the laboratory work and field sampling and wrote the first draft of the manuscript. TM and DS analyzed and visualized the data. All authors interpreted the results, and edited, reviewed and revised the manuscript.

\section{FUNDING}

Open Access funding enabled and organized by Projekt DEAL.

\section{COMPETING INTERESTS}

The authors declare no competing interests.

\section{ADDITIONAL INFORMATION}

Supplementary information The online version contains supplementary material available at https://doi.org/10.1038/s43705-021-00086-0.

Correspondence and requests for materials should be addressed to Rolf Daniel.

Reprints and permission information is available at http://www.nature.com/ reprints

Publisher's note Springer Nature remains neutral with regard to jurisdictional claims in published maps and institutional affiliations.

Attribution 4.0 International License, which permits use, sharing, adaptation, distribution and reproduction in any medium or format, as long as you give appropriate credit to the original author(s) and the source, provide a link to the Creative Commons license, and indicate if changes were made. The images or other third party material in this article are included in the article's Creative Commons license, unless indicated otherwise in a credit line to the material. If material is not included in the article's Creative Commons license and your intended use is not permitted by statutory regulation or exceeds the permitted use, you will need to obtain permission directly from the copyright holder. To view a copy of this license, visit http://creativecommons. org/licenses/by/4.0/.

(c) The Author(s) 2022 